\title{
AM MODE: Using AM and FM Synthesis for Acoustic Drum Set Augmentation
}

\author{
Cory Champion \\ New Zealand School of Music \\ Victoria University of Wellington \\ New Zealand \\ cory.champion@gmail.com
}

\author{
Mo H. Zareei \\ New Zealand School of Music \\ Victoria University of Wellington \\ New Zealand \\ mo.zareei@vuw.ac.nz
}

\begin{abstract}
AM MODE is a custom-designed software interface for electronic augmentation of the acoustic drum set. The software is used in the development a series of recordings, similarly titled as AM MODE. Programmed in Max/MSP, the software uses live audio input from individual instruments within the drum set as control parameters for modulation synthesis. By using a combination of microphones and MIDI triggers, audio signal features such as the velocity of the strike of the drum, or the frequency at which the drum resonates, are tracked, interpolated, and scaled to user specifications. The resulting series of recordings is comprised of the digitally generated output of the modulation engine, in addition to both raw and modulated signals from the acoustic drum set. In this way, this project explores drum set augmentation not only at the input and from a performative angle, but also at the output, where the acoustic and the synthesized elements are merged into each other, forming a sonic hybrid.
\end{abstract}

\section{Author Keywords}

Augmented drum set, Max/MSP, modulation synthesis, improvisation, live electronics.

\section{CCS Concepts}

-Applied computing Sound and music computing - Applied computing $\sim$ Performing arts $\bullet$ Information systems $\sim$ Music retrieval

\section{BACKGROUND}

AM MODE primarily revolves around seamless fusion of the synthesized and the acoustic. This approach echoes the first author's interest in the canonical relationship between the studio experimentation of jazz-fusion musicians of the early 1970s and elements of form, sonority, and synthesis employed in Detroit Techno from the mid 1980s onwards [1]. In his book More Brilliant Than The Sun: Adventures In Sonic Fiction, Kodwo Eshun defines this relationship by creating an idea of the Futurhythmachine:

At the Century's End, the Futurhythmachine has 2 opposing tendencies, 2 synthetic drives: the Soulful and the Postsoul. But then all music is made of both tendencies running simultaneously at all levels, so you can't merely oppose a humanitarian $\mathrm{r} \& \mathrm{~b}$ with a posthuman Techno [2].

The Futurhythmachine is a paradoxical neologism that Eshun applies to the dissection of rhythmic language modes of a spectrum of afrofuturist music. It is a conceptual framework for constructing the modulation systems, and a driving aesthetic force for the compositions.

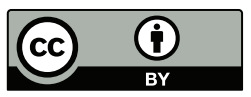

Licensed under a Creative Commons Attribution 4.0 International License (CC BY 4.0). Copyright remains with the author(s)

NIME'18, June 3-6, 2018, Blacksburg, Virginia, USA.

\section{RELATED WORKS}

There are numerous instances and applications of the augmented drum set within scholarly research, as well as commercially available technology. Beyond the bell and block ornamentations of Baby Dodds' Spooky Drums (1946), there are examples of electronic amplification of the drum set on the free improvisation axis on by Tony Oxley and Paul Lytton that employ pickup and amplification techniques derivative of the work of Karlheinz Stockhausen in Microphonie 1 (1964).

With the development of recording technology came the new ability to sonically augment the drum set post recording, such as the prototypical futurhythmachines of Jabali Billy Hart's modulated hihats on Herbie Hancock's Sextant (1973) and Teo Macero's filtered, phased, and spatial destruction of the drums in Miles Davis' music of the early 1970s. With the further development and ubiquity of analog synthesis technology came an expansion of early commercial percussion synthesizer effects to entire electronic drum sets of Simmons [3], followed by MIDI and sampling technology in the 1980s, which led to the digital electronic drum set giving performers the ability to store and playback any recorded sound.

Chris Cutler is drummer who has experimented with the 'electrification' of the drum set since the 1970s and summarizes the limitations of sample playback with the drums, a seemingly frictionless technology: "Pads are just a complementary system that sits alongside - or replaces - an acoustic drum. There is no hybridization, mutation or evolution, just two wholly different systems running in parallel" [4].

The music of Deantoni Parks highlights solo improvisation through limitations of playing a keyboard sampler with one hand and a drum-kit with the other simultaneously (e.g. Technoself, 2015). The sampled material informs the performance of both drums and the sampler, and while the execution is precise and the music is focused and minimal, the acoustic properties of the drum set are not augmented.

As technology has developed further, so has the capacity of sampling, and the complexity of control interfaces. Sensory Percussion by Sunhouse ${ }^{1}$ uses custom software and triggers with DSP to create a deeply expressive system for audio integration with acoustic drums. Further research over the past decade includes the EMdrum [5], Roberto Aimi's real-time convolution hybrid percussion instruments [6], and Christos Michalakos' The Augmented Drum Kit [7], which uses physical gestures performed on the drum set to manipulate electronic sound and light in real time. The drums are electronically resampled, re-pitched and delayed through a custom-built granular synthesis patch in Max/MSP.

Building upon this rich body of work, this project approaches drum set augmentation from an aesthetically stripped down and reductionist angle. In doing so, the developed toolkit is

\footnotetext{
${ }^{1} \mathrm{https://sunhou.se/}$
} 
consciously restricted to pure sinewaves and basic modulation techniques, AM and FM. Primarily a performer and an improviser, the first author has developed this project as a vehicle for solo improvisation, employing this reductionist approach and limited sonic palette as a compositional tool in interpreting Eshun's idea of the Futurhythmachine.

The next section summaries the development of this project. A brief technical overview of the system is outlined, followed by a discussion on the compositional process and resulting artwork.

\section{AM MODE}

In order to capture the input data from the drum set, two Roland drum triggers were used; one trigger on the bass drum and the other freely between snare drum, rack tom, and floor tom at the user's discretion. A combination of dynamic and condenser microphones was used, both for control input and for the recording of the drum set signal.

\subsection{System overview}

The software is developed as custom modulation units, each created in a patcher object in Max/MSP (see Figure 1). The custom object modeam $\sim$ uses cycle $\sim$ to modulate the amplitude of an audio signal, while the custom object modefm $\sim$ uses a cycle to modulate the frequency of a carrier cycle . The sinusoid waves of modefm $\sim$ are the sole synthetic sounds of the compositions, and the arguments for these objects are prepared and defined by the acoustic drum performance.

freakint $\sim$ is an adaptation of Miller Puckette's sigmund $\sim^{2}$ that detects the dominant frequency of an analog input as an integer value. This is employed to define the carrier oscillator's center frequency using the pitch of the acoustic drum. To add dynamic expression to the modulation unit, a custom sub-patcher called perctrig $\sim$ was created. Using Roland drum triggers, perctrig outputs a scaled integer related to the velocity at which the drum is struck.

The synthesized output signals of AM and FM units are controlled using a custom-designed envelope follower titled smartenv $\sim$. This unit helps track the amplitude of the live drum input using a simple AR envelope and a low pass filter.

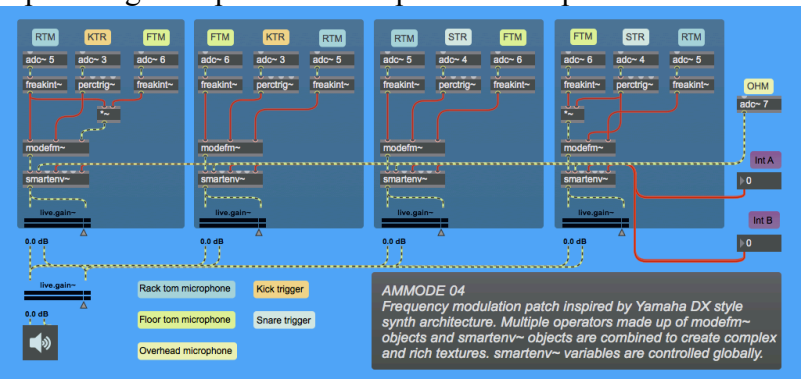

Figure 1. One of the used patch configurations

In order to enable independent modulation of the individual instruments, longer acoustic textures of the drum set and slower and more 'spacious' textures would need to be employed. While interacting with the system, some considerations are the tuning of the drums, the overall volume, the resonances space the drums are recorded, the use of sticks, mallets, or brushes, the use of drum rolls, and the placement of the microphones.

\subsection{Artistic Output}

AM MODE was used in the creation of series of seven recordings, titled $A M M O D E$. Throughout the entire album, every piece was initially improvisatory in its conception and was realized empirically, upon hours of experimentation. The compositional process was primarily informed through finding

\footnotetext{
${ }^{2} \mathrm{http} / / / \mathrm{www} . \max$ objects.com/?v=objects\&id_objet $=4713$
}

elements that best highlighted the desirable characters of the particular acoustic drum sound. This was accomplished by experimenting with variations in orchestration, texture, and dynamics, as well as different permutations of the synthesized output through exploring intricacies of the modulation networks unique to each improvisation. After hours of improvisation on a given software configuration, an arrangement of the composition was solidified and recorded live.

The recordings portray a wide variety of textures, nuances and dynamic ranges that are embedded in each performance. In this way, the work straddles the dichotomy of Eshun's Futurhythmachine, as it articulates interesting relationships between seemingly contrasting, yet fundamentally related, musical styles (jazz/techno), practices (improvisation/live electronics), and mediums (acoustic /synthesized).

$A M M O D E$ was self-released and can be accessed online at http://actualbone.bandcamp.com. A video demonstration of the first author interacting with the system can be viewed at https://youtu.be/7a3dmnZ3lTo.

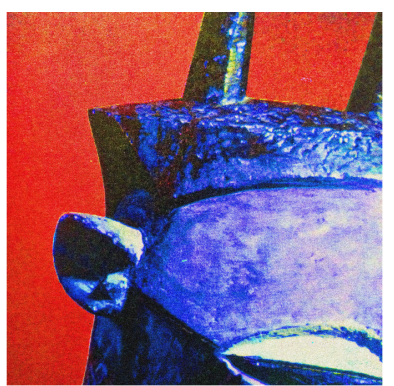

Figure 2. $A M M O D E$ : album art

\section{FUTURE WORK}

Improvisation is an essential and inherently integrated aspect of this project. Therefore, as was observed throughout this project, in its current state, the software is more suitable for recording improvisations. However, more avenues could be explored with regard to collaboration. Having a user/performer controlling the interface, while another playing the drums in a live context can lead to exciting possibilities. Additionally, and with regard to the programming, further development can be done in order to achieve a more modular and customizable design, where any potential user can modify the signal flow and modulation unit routings, and adapt the system to their needs and desires.

\section{REFERENCES}

[1] S. Goldmann. Mills, Hood and FM Synthesis as a Metaphor, The Wire \#377 (July 2015).

[2] K. Eshun. More Brilliant Than The Sun: Adventures in Sonic Fiction. London: Quartet Books, 1998.

[3] D. Simmons, Dave Simmons and the Simmons Story. Available online at www.simmonsdrums.net/dave-simmons-story/ (accessed April 2018).

[4] C. Cutler, The Electrified Kit. available online at: http://www.ccutler.com/ccutler/electrifiedKit.shtml (accessed April 2018).

[5] D. Rector, S. Topel. EMdrum: An Electronically Actuated Drum. In Proceedings of the 2014 International Conference on New Interfaces for Music Expression (NIME). (London, UK).

[6] R. Aimi. Percussion instruments using realtime convolution: Physical controllers. In Proceedings of the 2007 Conference on New Interfaces for Music Expression (NIME) (New York, NY, USA).

[7] C. Michalakos. The Augmented Drum Kit: An Intuitive Approach to Live Electronic Percussion Performance. In Proceedings of the International Computer Music Conference (ICMC (Ljubljana September 2012). 